\title{
A STRENGTHENING OF LETH'S UNIQUENESS CONDITION FOR SEQUENCES
}

\author{
JEROME MALITZ
}

\begin{abstract}
A series $\sum a_{i}$ of nonnegative reals summing to 1 such that $a_{i} \leq$ $\sum_{j>i} a_{j}$ for each $i$ is uniquely characterized by the equalities of the form $\sum_{\text {Leth. }} a_{i}=\sum_{K} a_{k}$. This characterization is an improvement of one given by
\end{abstract}

The main purpose of this note is to prove the following sharpened version of a theorem of S. Leth [2].

THEOREM. Let $\left\langle a_{n}\right\rangle$ and $\left\langle b_{n}\right\rangle$ be sequences of real numbers such that

(i) $\lim _{n \rightarrow \infty} a_{n}=\lim _{n \rightarrow \infty} b_{n}=0$,

(ii) $0<a_{n+1} \leq a_{n}$ and $0<b_{n+1} \leq b_{n}$ for all $n$,

(iii) $a_{n} \leq \sum_{j>n} a_{j}$ and $b_{n} \leq \sum_{j>n} b_{j}$ for all $n$,

(iv) $\sum_{j \in J} a_{j}=\sum_{k \in K} a_{k}$ iff $\sum_{j \in J} b_{j}=\sum_{k \in K} b_{k}$ for all $J$ and $K$.

Then there is a constant $u$ such that $a_{i}=u b_{i}$ for all $i$.

In Leth's theorem (iv) is replaced by

(iv) $^{\prime} \sum_{j \in J} a_{j} \leq \sum_{k \in K} a_{k}$ iff $\sum_{j \in J} b_{j} \leq \sum_{k \in K} b_{k}$ for all $J$ and $K$.

J. Mycielski [3] asked if (iv) suffices. To see that the answer to his qustion is yes, we need several lemmas. The lemmas and their proofs are variants of those in [1 and 2]. $N$ is the set of nonnegative integers.

LEMMA 1. Let $r \leq \sum_{i=1}^{\infty} a_{i}$ where $a_{n+1} \leq a_{n} \leq \sum_{j>n} a_{j}$ for all $n$ and $\lim _{n \rightarrow \infty} a_{n}=0$. Then there is some $K \subseteq N$ such that $r=\sum_{K} a_{k}$.

ProOF. We define $K=\left\{k_{0}, k_{1}, \ldots\right\}$ inductively. Let $k_{0}$ be the least $j$ such that $a_{j} \leq r$. If $k_{i}$ is known for $i<n$ and $\sum_{i<n} a_{k_{i}}<r$ let $k_{n}$ be the least $j$ such that $a_{j}+\sum_{i<n} a_{k_{i}} \leq r$ (such a $j$ exists since $\lim _{n \rightarrow \infty} a_{n}=0$ ); otherwise take $K=\left\{k_{0}, \ldots, k_{n-1}\right\}$. Clearly $\sum_{k \in K} a_{k} \leq r$. To see that we cannot have $\sum_{k \in K} a_{k}<r$ first note that the definition of $K$ and the assumption $\lim _{n \rightarrow \infty} a_{n}=0$ imply $K$ is infinite. By assumption $\sum_{k \in N} a_{k} \geq r$, hence $K \neq N$ and so there is a greatest $l \notin K$. But $a_{l} \leq \sum_{k>l} a_{k}$ forcing $l \in K-$ a contradiction. Therefore $\sum_{k \in K} a_{k}=r$.

LEMMA 2. Under the assumptions of Lemma 1 , for every $j$ there is a $K \subseteq$ $\{i: i>j\}$ such that $a_{j}=\sum_{K} a_{i}$. Hence $a_{j}=\sum_{L} a_{l}$ for some infinite $L$.

PROOF. This follows from Lemma 1 by considering the sequence $\left\langle a_{j+1+i}\right\rangle_{i=0}^{\infty}$ and taking $r$ to be $a_{j}$. Iterating this procedure on the last term of the expansion as long as the expansion is finite gives the desired infinite expansion.

Received by the editors October 15, 1985.

1980 Mathematics Subject Classification (1985 Revision). Primary 60A05; Secondary 60B99. 
LEMMA 3. Under the assumptions of Lemma 1 , for every $J \neq N$ there is a $K$ such that $\sum_{J} a_{j}=\sum_{K} a_{k}$ and $N-K$ is infinite.

ProOF. If $N-J$ is finite with largest member $j$, let $a_{j}=\sum_{P} a_{i}$ where $P$ is infinite as in Lemma 2. Now let $K$ be the complement of $((N-J)-\{j\}) \cup P$.

PROOF OF THE THEOREM. We show that (i)-(iv) imply (iv)'. If not, then there is a $J$ and an $L$ such that $\sum_{L} a_{l}<\sum_{J} a_{j}$ and $\sum_{L} b_{l}>\sum_{J} b_{j}$. Fix $J$ and let $r=\sup \left\{\sum_{L} b_{l}: \sum_{L} a_{l}<\sum_{J} a_{j}\right.$ and $\left.\sum_{L} b_{l}>\sum_{J} b_{j}\right\}$. By Lemma 1 there is an $M$ such that $\sum_{M} b_{m}=r$.

We claim that $\sum_{M} a_{m}<\sum_{J} a_{j}$. For let $M^{\prime}$ be a finite initial subset of $M$. Take $L$ such that $\sum_{L} a_{l}<\sum_{J} a_{j}, \sum_{L} b_{l}>\sum_{J} b_{j}$, and $\sum_{L} b_{l}>\sum_{M^{\prime}} b_{m}$. By Lemma 1 (with $r=\sum_{L} b_{l}-\sum_{M^{\prime}} b_{m}$ and $\sum_{i=1}^{\infty} a_{k}$ replaced by $\sum_{i>k} a_{i}$ where $k=\max M^{\prime}$ ) there is some $M^{\prime \prime}$ such that $M^{\prime} \subseteq M^{\prime \prime}$ and $\sum_{M^{\prime \prime}} b_{m}=\sum_{L} b_{l}$. Hence $\sum_{M^{\prime}} a_{m} \leq \sum_{M^{\prime \prime}} a_{m}=\sum_{L} a_{l}<\sum_{J} a_{j}$. Therefore $\sum_{M} a_{l} \leq \sum_{J} a_{j}$. Equalilty implies $\sum_{M} b_{j}=\sum_{J} b_{j}$ by (iv), so we must have $\sum_{M} a_{m}<\sum_{J} a_{j}$, as claimed.

By Lemma 3, we may assume that $N-M$ is infinite and since $\lim _{n \rightarrow \infty} a_{n}=0$, there is a $j \in N-M$ such that $a_{j}+\sum_{M} a_{m}<\sum_{J} a_{j}$. But then $b_{j}+\sum_{M} b_{m}>r$ and also $b_{j}+\sum_{M} b_{m}>\sum_{J} b_{j}$, a contradiction which finishes the proof.

\section{REFERENCES}

1. R. Chauqui and J. Malitz, Preorderings compatible with probability measures, Trans. Amer. Math. Soc. 279 (1983), 811-824.

2. S. Leth, $A$ uniqueness condition for sequences, Proc. Amer. Math. Soc. 93 (1985), 287-290.

3. J. Mycielski, Personal communication.

Department of Mathematics, University of Colorado, Box 426, Boulder, COLORADO 80309 